Please quote as: Knop, N., Blohm, I. \& Leimeister, J. M. (2019): Internes Crowdsourcing - Herausforderungen und Lösungsstrategien für eine erfolgreiche Transformation der Arbeitsorganisation. In: HMD Praxis der Wirtschaftsinformatik. Wiesbaden, Germany. 


\title{
Internes Crowdsourcing - Herausforderungen und Lösungsstrategien für eine erfolgreiche Transformation der Arbeitsorganisation
}

\author{
Nicolas Knop $(\mathbb{D} \cdot$ Ivo Blohm • Jan Marco Leimeister
}

Eingegangen: 11. März 2019 / Angenommen: 20. Juni 2019

(C) Springer Fachmedien Wiesbaden GmbH, ein Teil von Springer Nature 2019

Zusammenfassung Dieser Artikel hilft Organisationen ihre Mitarbeiter mittels Crowdsourcing in einem digitalen Umfeld zu reorganisieren, um Agilität, Produktivität und Effektivität der Geschäftsprozesse zu erhöhen. Internes Crowdsourcing, in welchem die kollektive Arbeitskraft, Kreativität und Intelligenz der Mitarbeiter effektiver genutzt wird, stellt eine flexible, dezentrale sowie digitale Form der Arbeitsorganisation dar. Dieser Prozess resultiert in einer Transformation der Organisation, der über die einfache Einrichtung der Crowdsourcing-Plattform hinausgeht. Aus diesem Grund stehen viele Unternehmen vor Problemen, diesen Transformationsprozess erfolgreich zu gestalten und können die Vorteile von Crowdsourcing nicht oder nur eingeschränkt nutzen. Daher identifizieren wir in unserem Artikel, basierend anhand einer multiplen Fallstudie fünf Herausforderungen und dazu passende Maßnahmen, um eine erfolgreiche Transformation zu managen. In diesem Rahmen gehen wir in einem Fall auf die Herausforderungen und Lösungen im Detail ein, auf denen wir dann in den zusätzlichen Fällen aufbauen. Schließlich präsentieren wir fünf Herausforderungen, wie inadäquate IT Fähigkeiten und die passenden Lösungsansätze, wie gezielte Schulungen. Diese erlauben es Unternehmen Internes Crowdsourcing als eine innovative Form der Arbeitsorganisation zu nutzen und Agilität sowie Effektivität interner Arbeitsprozesse zu erhöhen.

Schlüsselwörter Internes Crowdsourcing - Transformationsherausforderungen · Crowdsourcing-Plattform · Crowdtesting · Multiple Fallstudie

\footnotetext{
N. Knop $\cdot$ I. Blohm $\cdot$ J. M. Leimeister $(\bowtie)$

Institut für Wirtschaftsinformatik, Universität St. Gallen, Müller-Friedbergstraße 8, 9000 St. Gallen, Schweiz

E-Mail: janmarco.leimeister@unisg.ch
} 


\title{
Internal Crowdsourcing - Challenges and Solutions for a Successful Transformation of Work Organizations
}

\begin{abstract}
This article helps organizations to reorchestrate their employees by using the principles of crowdsourcing to increase the agility, productivity and effectiveness of their business operations in an increasingly digital environment. Internal crowdsourcing in which the collective workforce, creativity, and intelligence of employees is harnessed, reflects an innovative, decentralized and flexible form of digital work organization. These major organizational processes transform the company and, thus, go beyond setting up a crowdsourcing platform for employees. Many companies struggle to manage this transformation process successfully and cannot capture the benefits of internal crowdsourcing effectively. Based on a multiple case study approach, this paper in hand identifies five transformation challenges and corresponding countermeasures for mastering the transformation described. We analyze the transformation challenges and countermeasures in depth in one case and verify the findings in two additional cases. Finally, the paper gives insights on what challenges might occur, provides opportunities to identify them early and counter them successfully. Finally, we present five challenges, such as inadequate IT capabilities and appropriate solutions, such as specific trainings. These enable companies to apply internal crowdsourcing as new form of work orchestration increasing agility and process efficiency.
\end{abstract}

Keywords Internal Crowdsourcing - Transformation Challenges · CrowdsourcingPlatform · Crowdtesting · Multiple Case Study

Digitale Technologien haben es Unternehmen ermöglicht, die Kreativität, die verteilte Arbeitskraft und das Wissen einer großen Anzahl von Individuen systematisch zu nutzen. Crowdsourcing hat sich in einer Vielzahl von Anwendungskontexten fest etabliert, um Probleme schneller, besser und günstiger zu lösen. Heute nutzen bereits $84 \%$ aller Fortune 500 Unternehmen Crowdsourcing, um eine große Anzahl von Internetnutzern in ihre Wertschöpfungsprozesse zu integrieren.

In den vergangenen Jahren erfreute sich ,internes Crowdsourcing“ zunehmender Beliebtheit (Zuchowski et al. 2016). Im Gegensatz zu externem Crowdsourcing, formt sich die teilnehmende Crowd nicht aus anonymen Internetnutzern, sondern aus Mitarbeitern einer Organisation. Der Grundgedanke ist, dass ein Unternehmen seine Mitarbeiter bzw. eine bestimmte Gruppe von Mitarbeitern einlädt, eine Aufgabe freiwillig über eine IT-Plattform zu lösen (Durward et al. 2016). Internes Crowdsourcing kann zur Unterstützung einer Vielzahl unterschiedlicher Geschäftsprozesse eingesetzt werden (Simula und Vuori 2012), z. B. der Innovations- (Benbya und Leidner 2016) und Strategieentwicklung (Stieger et al. 2012) oder dem Erstellen von Marktvorhersagen (Rohrbeck et al. 2015). Vor diesem Hintergrund planen viele Unternehmen, bestimmte Aufgaben systematisch von einem flexiblen Pool interner Crowd Worker bearbeiten zu lassen (Kohler 2015). Zum Beispiel hat Dieter Zetsche - der Vorstandsvorsitzende der Daimler AG - angekündigt, 20\% des Personals für 
eine Reihe von Innovationsaufgaben in eine interne Crowd umzuwandeln (Zetsche 2017).

Die Ausgestaltung und der Aufbau solch einer großen internen Crowd umfasst jedoch einen erheblichen organisatorischen Transformationsprozess, den es zu managen gilt (Malhotra und Majchrzak 2014). Wie langwierig dieser Wandel sein kann, hat hierbei Allianz UK festgestellt, als eine interne Crowdsourcing-Plattform zum Zwecke der Innovationsentwicklung eingeführt wurde. Von der Inbetriebnahme bis zur effizienten Nutzung der Plattform vergingen mehr als acht Jahre. Die zu überwältigenden Herausforderungen umfassten dabei nicht nur das Aufbauen und Verstetigen einer ausreichend großen internen Crowd, sondern auch die Umstellung der kompletten Arbeitsorganisation von oftmals hierarchischen und formalen Arbeitsprozessen hin zu einer dezentralisierten Organisation mit egalitären Kommunikationsstrukturen (Benbya und Leidner 2016). Ziel des folgenden Beitrags ist es, Unternehmen dabei zu unterstützen, die mit der Einführung von internem Crowdsourcing einhergehenden Transformationsprozesse erfolgreich zu meistern. Vor diesem Hintergrund berichten wir in Fallstudien über drei Unternehmen, die internes Crowdsourcing eingeführt haben. Es werden fünf übergreifende Transformationsherausforderungen skizziert und entsprechende Lösungsstrategien aufgezeigt.

\section{Die Fallstudien}

Zur Identifikation der mit der Einführung von internem Crowdsourcing einhergehenden Herausforderungen und Lösungsansätze haben wir drei Fallstudien durchgeführt (siehe Tab. 1). Wir haben erforscht, wie Unternehmen aus unterschiedlichen Branchen - Finanzdienstleistungen und verarbeitendes Gewerbe - die mit internem Crowdsourcing einhergehenden Transformationsherausforderungen bewältigen (Yin 1994). Da sich über alle drei Fallstudien hinweg ein sehr konsistentes Bild abzeichnete, fokussieren wir im Rahmen des Beitrages auf die „Bank AG“. Dadurch können wir im Detail auf organisatorische Rahmenbedingungen, die Art und Weise des systematischen Einsatzes von internem Crowdsourcing und die damit einhergehenden Transformationserfordernisse eingehen. Diese Beschreibung werden durch erfolgreiche Lösungsansätze aus den beiden anderen Fallstudien - der „Versicherungen AG“ und der „Industrie AG“ ergänzt, um ein besser generalisierbares Bild zu zeichnen.

Um zwischen den Fallstudien vergleichbare Ergebnisse zu gewährleisten, haben wir uns auf Unternehmen konzentriert, die internes Crowdsourcing in einem vergleichbaren Setting einsetzen - Software Testing. Das Testen von Software ist eine komplexe Aufgabe, die sowohl Fach- als auch Domänenwissen benötigt und die allen Software-Applikationen regelmässig durchgeführt werden muss (Leicht et al. 2017). Vor diesem Hintergrund ist Software Testing als Regeltätigkeit ein gut geeigneter Untersuchungskontext, um die Einführung von internem Crowdsourcing als neue Form der Arbeitsorganisation zu untersuchen.

In den Fallstudien haben wir zwischen September 2016 und März 2018 verschiedene Datenquellen genutzt (Creswell 1998; Eisenhardt 1989). Diese umfassen 19 Interviews von ca. 40-80 min, in denen die Herausforderungen und Lösungen der Transformation diskutiert werden. Weitere Quellen umfassen aktive Beobachtungen 
Tab. 1 Untersuchte Fallstudien

\begin{tabular}{|c|c|}
\hline Fall & Beschreibung \\
\hline Bank AG & $\begin{array}{l}\text { Die Bank AG ist eine der traditionsreichsten Retail-Banken der Schweiz. Das Unterneh- } \\
\text { men baute eine interne Crowd aus Mitarbeitern aus den einzelnen Filialen und Mitarbei- } \\
\text { tern auf, um eine geschäftskritische Software-Applikation zu testen, die von nahezu jedem } \\
\text { Mitarbeiter mehrmals täglich genutzt wird. Internes Crowdsourcing ist heute Teil des } \\
\text { Standard-Testprozesses für diese Applikation }\end{array}$ \\
\hline $\begin{array}{l}\text { Versiche- } \\
\text { rungen } \\
\text { AG }\end{array}$ & $\begin{array}{l}\text { Die Versicherungen AG ist einer Schweizer Versicherungsgesellschaft mit Fokus auf } \\
\text { Krankenversicherungen. Aufbauend auf einem erfolgreichen Pilottest für internes Crowd- } \\
\text { testing wurde eine interne Dienstleistung aufgesetzt, mit der IT-Projekte systematisch auf } \\
\text { eine interne Crowd aus Mitarbeitern zum Software-Testing zugreifen können }\end{array}$ \\
\hline $\begin{array}{l}\text { Industrie } \\
\mathrm{AG}\end{array}$ & $\begin{array}{l}\text { Die Industrie AG ist ein Schweizer Industrieunternehmens mit einem globalen Netzwerk } \\
\text { aus Landesgesellschaften. Internes Crowdsourcing wird hier eingesetzt, um die einzelnen } \\
\text { Landesgesellschaften in den Prozess des Software Testing einzubinden }\end{array}$ \\
\hline
\end{tabular}

bei den Fallstudienpartnern. Darüber hinaus analysierten wir erstellte Projektdokumentation der Fallstudienpartner, sowie die auf den Crowdtesting-Plattformen angefallenen Daten aus den einzelnen Tests. Bei der Bank AG wurde zudem eine Umfrage von 109 Mitarbeitern über die Akzeptanz von Crowdsourcing als neue Form Arbeitsorganisation durchgeführt.

\section{Internes Crowdsourcing als Transformationsprozess bei der Bank AG}

Im Folgenden stellen wir die interne Crowdsourcing-Initiative der Bank AG vor. Dabei beschreiben wir, wie Arbeitsprozesse in einem großen und unternehmensweiten Software Testing Projekt mittels internem Crowdsourcing restrukturiert wurden und gehen auf den damit verbundenen Transformationsprozess ein.

\subsection{Ausgangslage und Ziele}

Die Bank AG ist eine führende Schweizer Retail-Bank mit über 9000 Mitarbeitern und 3,7 Mio. Kunden und einer Geschäftstätigkeit in der gesamten Schweiz. Die Bank entwickelte eine neue geschäftskritische Unternehmenssoftware, die von fast jedem Mitarbeiter täglich genutzt wird. Bereits während des Entwicklungsprozesses erkannte die Projektleitung, dass die zu Verfügung stehenden Ressourcen für das Testen der Software nicht ausreichen werden - trotz eines Projektteams von mehreren hundert Personen. Die Bank AG lief damit nicht nur Gefahr die eigenen Ziele hinsichtlich Qualität und Verwendbarkeit der Software zu unterlaufen, sondern auch regulatorische Anforderungen nicht zu erfüllen. So begann die Bank AG ab Ende 2016 mit der Einführung des internen Crowdsourcing, um die Endnutzer der Software, d.h. die Bank- und Filialmitarbeiter, in einem ausgedehnten „Crowdsourced Software Testing (Crowdtesting)“ zu integrieren.

Anstelle ausgewählter Mitarbeiter zum Softwaretest in die Zentrale einzuladen, führte die Bank eine interne Crowdsourcing-Plattform ein, um die Einbindung von Endnutzern in das Software Testing zu skalieren. Dadurch konnten nicht nur Reisekosten und Arbeitszeit der teilnehmenden Tester reduziert, sondern auch die An- 
zahl der eingebundenen Mitarbeiter beträchtlich gesteigert werden. Zudem wurde es dadurch möglich Endnutzer nicht nur in finale Akzeptanztests zu involvieren, sondern bereits in die frühen Phasen von Softwareentwicklungsprojekten, in denen Testbedürfnisse häufig Ad-hoc und unvorhergesehen anfallen. Bei der Software handelt es sich um eine branchenspezifische Standardlösung, die eine umfassende Anpassung an die Geschäftsprozesse, Produkte/Dienstleistungen und umliegenden IT-Systeme der Bank AG erforderte. Potenzielle Software-Tester benötigten daher fundierte Kenntnisse der Geschäftsabläufe und Produkte der Bank - Kenntnisse, die sich über eine Vielzahl von unterschiedlichen Abteilungen verteilten und eine Form der Vernetzung der verschiedenen Wissensträger erforderten.

Durch internes Crowdsourcing wollte die Bank AG nicht nur die Software-Qualität steigern, sondern auch den Einsatz von Endnutzern im Testing beschleunigen und flexibilisieren. Dabei war es jedoch nicht das Ziel, das internes Crowdtesting das traditionelle Software-Testing ersetzt. Vielmehr wurde es als Ergänzung eingesetzt, um kollektives Wissen und Arbeitskraft der Mitarbeiter zu bündeln.

\subsection{Durchführung des internen Crowdtesting}

Die Bank AG baute eine Crowd von 216 Mitarbeitern auf, welche die Software über einen Zeitraum von 15 Monaten testete. Die Crowd wurde hinsichtlich ihrer Expertise in die fünf Produktbereiche der Bank - z. B. „Kredite und Darlehen“ oder „Altersvorsorge“ - segmentiert. Dadurch wurde sichergestellt, dass einzelne Tester über genügend Geschäftsexpertise verfügen, um die entsprechenden Funktionalitäten der Software richtig zu testen. Für jeden Produktbereich wurde ein wöchentlicher Testlauf durchgeführt, in dem ca. 15-20 Mitarbeiter eingesetzt wurden. In Summe testeten jede Woche durchschnittlich um die 90 Mitarbeiter die Software. Für das Management des gesamten Crowdtesting-Prozesses wurde ein dediziertes Team aufgebaut - das Crowdtesting Management Office (CMO). Das CMO diente als Schnittstelle zwischen der Crowd und den Software-Entwicklungsteams. Es half den einzelnen Entwicklungsteams ihre Testbedarfe „crowdsourcing-fähige“ Software-Testaufgaben zu überführen und war verantwortlich für das Aufsetzen der wöchentlichen Crowdtesting-Iterationen.

Der Crowdtesting-Prozess besteht aus drei allgemeinen Schritten:

1. Basierend auf den spezifischen Testbedarfen stellt das CMO aus dem Pool der verfügbaren Tester eine angemessene Anzahl von Mitarbeitern für den jeweiligen Test zusammen. Die Teilnahme der Crowd war freiwillig und erfolgte während der regulären Arbeitszeit. Sie sollten zwischen einem und einem halben Arbeitstag testen. Aus Compliance-Gründen wurden der Crowd keine finanziellen Belohnungen für die Teilnahme angeboten.

2. Der eigentliche Softwaretest wurde über eine IT-Plattform durchgeführt, die Zugang zu den Testaufgaben ermöglicht sowie eine Eingabemaske zur Dokumentation von Softwarefehlern und anderen zu behebenden Problemen bot. Zur Unterstützung des Testing wurden verschiedene Kommunikationskanäle eingesetzt. Zum einen wurde es der Crowd ermöglicht, sich während den einzelnen Tests direkt auszutauschen und anstehende Probleme zu diskutieren. Zum anderen gab es 
einen zentralen Kommunikationskanal, mit dem das CMO Informationen an allen beteiligten Tester gleichzeitig übermitteln konnte. Während der Tests wurde die Crowd von erfahrenden Kollegen begleitet, um die Crowd bei ihren Testaufgaben zu unterstützen. In der Regel waren dies ,,professionelle“ Tester und Entwickler aus dem Projekt.

3. Schließlich bewerteten die technischen Experten und das CMO nach den Tests alle durch die Crowd dokumentierten Fehler und Softwareprobleme. Verifizierte Fehler und Softwareprobleme wurden dann an den Priorisierungsprozess des Softwareentwicklungsprojekts weitergeleitet, von wo aus sie in die weitere Entwicklungs-Roadmap aufgenommen wurden.

\subsection{Veränderungen in der Arbeitsorganisation}

Für viele Mitarbeiter der Bank AG war das interne Crowdsourcing eine neue Arbeitserfahrung, so dass es eine Änderung ihrer konkreten Arbeitsweise erforderte. In traditionellen Arbeitsumgebungen befinden sich die Mitarbeiter in der Regel in klaren und gut organisierten hierarchischen Strukturen innerhalb einer bestimmten Geschäftseinheit oder Abteilung. Ein direkter Vorgesetzter ist verantwortlich und kann per Weisung einzelne Mitarbeiter steuern. Mitarbeiter kennen und arbeiten mit den meisten Kollegen sowie ihre direkten Vorgesetzten persönlich zusammen. Im Gegensatz dazu waren die Kollegen im digitalen Arbeitsumfeld des internen Crowdsourcing nur online und remote verfügbar. Zu Beginn des Projektes mussten die Mitarbeiter der Bank AG lernen, wie sie sich in dieser neuen Arbeitsumgebung bewegen und interagieren müssen. Die meisten Crowd Worker stammten aus einem Tätigkeitsbereich ohne direkten IT-Bezug und mussten daher in einem digitalen Setting die komplexe Aufgabe des Software-Testens erlernen. Gerade zu Beginn des Projektes mussten sich die Teilnehmer, an die neue Form der Arbeitsorganisation gewöhnen - dem offenen Aufruf zur freiwilligen Teilnahme am Software Testing. Für Verwirrung sorgte dabei insb. zu Anfang die für viele Crowd Worker schwer nachvollziehbare Aufgaben, Verantwortlichkeiten und Kompetenzen. So stammte der Großteil der Teilnehmer aus Organisationsstrukturen, in denen Ihre Aufgaben fest definiert wurden und ihnen direkt zur Bearbeitung zugewiesen wurden. Im Gegensatz dazu sollten die Crowd Worker sich selbständig entscheiden an den Crowdtests teilzunehmen und ihre Aufgaben im Detail zu definieren.

\subsection{Zentrale Ergebnisse}

Trotz der herausfordernden Veränderungen in der Arbeitsorganisation integrierte die Bank AG erfolgreich 216 Mitarbeiter in das Software Testing, die 14 monatelang ca. 50 Crowd-Tests durchführte, was in ca. $7000 \mathrm{~h}$ Software-Testing und mehr als 5000 dokumentierte Software-Probleme einreichte. Ohne die umfassende Unterstützung der Crowd hätte die Bank die Entwicklung dieser großen Software, die heute von jedem Mitarbeiter in der täglichen Praxis eingesetzt wird, nicht durchführen können. 


\section{Herausforderungen bei der Einführung von Internem Crowdsourcing}

Im Rahmen der drei durchgeführten Fallstudien haben wir fünf übergeordnete Herausforderungen bei der Einführung von internem Crowdsourcing entdeckt (siehe Tab. 2).

- 1. Organisatorische Grenzen: Internes Crowdsourcing entspricht im weitesten Sinne dem Aufbauen eines flexiblen Ressourcen-Pools, der bei Bedarf genutzt werden kann - oftmals ohne definierte Hierarchien und Reporting-Strukturen. Während das Top-Management in den drei untersuchten Unternehmen grundsätzlich sehr offen gegenüber diesen Ideen war, konnten bei allen drei Unternehmen Spannungen auf der Ebene der direkten Vorgesetzten der teilnehmenden Mitarbeiter beobachtet werden. Das Abwandern der „eigenen“ Mitarbeiter in die interne Crowd wurde dabei teilweise als Verlust von Ressourcen und Macht angesehen - insbesondere, wenn internes Crowdsourcing während der regulären Arbeitszeiten durchgeführt werden sollte. Darüber hinaus können Arbeitnehmervertretungen sensibel auf die potenzielle Ausbeutung von Mitarbeitern sowie einer Verschlechterung der Arbeitsbedingungen reagieren und internes Crowdsourcing reglementieren bzw. unterbinden.

Im Fall der „Versicherungen $\mathrm{AG}^{\prime \prime}$ hat sich der Betriebsrat eingeschaltet um zu prüfen wie die gesamte Crowdsourcing-Initiative gestaltet werden sollte. Er stellte Fragen bezüglich der zeitlichen Beteiligung einzelner Mitarbeiter und wie Vorgesetze in den Prozess eingebunden würden. Der Betriebsrat wollte sicherstellen, dass einerseits die Arbeitnehmerseite die Möglichkeit bekommt, die Aufgaben während der Arbeitszeit zu absolvieren und anderseits, dass die Arbeitgeberseite die betriebliche Sicherheit, auch bei großem Zuspruch seitens der Belegschaft, jederzeit gewährleisten kann.

- 2. Gehypte Erwartungen: Viele Mitarbeiter haben gerade zu Beginn überzogene Erwartungen an die Teilnahme von internem Crowdsourcing - eine innovativere, demokratischere, autonomere und spannendere Arbeitsweise. Dies ist insbesondere eine große Herausforderung, wenn Crowd Worker eine ganz andere Vorstellung von internem Crowdsourcing und ihre eigentliche Aufgabe haben, als vom Unternehmen und dem CMO angedacht wurde. Eine solche Diskrepanz birgt zwangsläufig das Risiko negativer Überraschungen, die das Bild des internen Crowdsourcing nachhaltig bei den Crowd Workern prägen können und im schlimmsten Fall zu einer sinkenden Beteiligung und/oder schlechterer Qualität der erzielten Ergebnisse führen können.

So testete die Bank AG beispielsweise eine unternehmensweite Standardsoftware, die an die Anforderungen der Bank angepasst wurde. Als logische Konsequenz konnte die Funktionalität der Software nur in gewissen Leitplanken angepasst werden. Im Gegensatz dazu gingen viele Crowd Worker jedoch davon aus, dass die Software aufgrund ihres Feedbacks grundsätzlich angepasst werden könnte, so dass sie fälschlicherweise davon ausgingen, dass ihr Feedback nicht umgesetzt und geschätzt wurde. Es wurde von einigen Crowd-Workern angenommen, dass ihre Aufgabe in der freien Exploration einer innovativen Software und dem anschlie- 
Tab. 2 Herausforderungen bei der Einführung von internem Crowdsourcing

\begin{tabular}{ll}
\hline Herausforderungen & Beschreibung \\
\hline $\begin{array}{l}\text { 1. Organisatori- } \\
\text { sche Grenzen }\end{array}$ & $\begin{array}{l}\text { Das Crowdsourcing-Management muss sicherstellen, dass die Forderungen der } \\
\text { Vorgesetzten der Crowd Worker, das Top-Management und der Betriebsrat berück- } \\
\text { sichtigen }\end{array}$ \\
$\begin{array}{l}\text { 2. Gehypte Er- } \\
\text { wartungen }\end{array}$ & $\begin{array}{l}\text { Das Crowdsourcing-Management steht vor der Herausforderung, die Erwartungs- } \\
\text { entwicklung der Mitarbeiter vom Höhepunkt unzureichender Erwartungen am }\end{array}$ \\
$\begin{array}{l}\text { Anfang bis zur Realität des Crowdsourcing zu begleiten und steuern } \\
\text { Fähigkeiten }\end{array}$ & $\begin{array}{l}\text { Das Management muss die IT- und Workflow-Fähigkeiten der Mitarbeiter auf ein } \\
\text { zu ermöglichen }\end{array}$ \\
$\begin{array}{l}\text { 4. Schwache } \\
\text { Anreizstruktur }\end{array}$ & $\begin{array}{l}\text { Die Herausforderung besteht darin, dass die Anreizstruktur zu schwach ist, um die } \\
\text { mangelt und die Teilnahme der Crowd an Initiativen freiwillig ist }\end{array}$ \\
$\begin{array}{l}\text { 5. Parallelität der } \\
\text { Arbeitsabläufe }\end{array}$ & $\begin{array}{l}\text { Eine häufige Transformationsherausforderung der Mitarbeiter ist der Wechsel } \\
\text { zwischen dem analogen Arbeitsalltag und dem digital vermittelten Arbeitsumfeld } \\
\text { der internen Crowd, was den Stress erhöhen und anstrengend sein kann }\end{array}$ \\
\end{tabular}

Benden Geben von Feedback bezüglich der eigenen Wahrnehmungen und Gedanken läge. So wurden viele der anfänglich sehr positiven Erwartungen durch den stark reglementierten Rahmen und den repetitiven Aufgaben des Softwaretestings enttäuscht. Internes Crowdsourcing ist nicht in jedem Fall das Streben nach erfüllter Arbeit, sondern kann auch harte und kognitiv anspruchsvolle Arbeit umfassen.

Auf Basis der Erfahrungen der Bank AG und der beiden anderen Fallstudien ist festzuhalten, dass Unternehmen bei internem Crowdsourcing einen gewissen „Hype Cycle“ durchlaufen (Steinert und Leifer 2010). Unternehmen stehen in gewisser Weise vor der Herausforderung, teilnehmende Crowd Worker zunächst in Ihren aufgeblähten Erwartungen zu bremsen und diese anschließend durch ein Tal der Tränen zu führen, bevor diese realistischen Erwartungen an die Teilnahme von internem Crowdsourcing entwickelt haben.

- 3. Inadäquate IT Fähigkeiten: Internes Crowdsourcing stellt eine innovative und IT-basierte Form der Arbeitsorganisation dar. Für eine erfolgreiche Teilnahme der Crowd Worker, ist es daher essenziell, dass sie befähigt werden, die Crowdsourcing-Plattform zu bedienen und die übergeordneten Arbeitsprozesse zu verstehen. Die Herausforderung für Unternehmen ist hier insbesondere die große Diversität der teilnehmenden Mitarbeiter - so muss internes Crowdsourcing im Prinzip so gestaltet werden, dass alle Mitarbeiter teilnehmen können. Vor diesem Hintergrund müssen entsprechende Onboarding- und ggf. auch Ausbildungsformate geschaffen werden.

Zum Beispiel hatten einige Mitarbeiter der Bank AG geringe IT- und WorkflowFähigkeiten, die Ergebnisse einreichten, die nicht zu ihrem Arbeitsumfang gehörten und keinen Mehrwert lieferten. Diese Crowd Worker verstanden den elementaren Arbeitsablauf und das Ziel der Initiative nicht. Daher konnten sie keine wertvollen Beiträge zur internen Crowdsourcing-Initiative einreichen.

- 4. Schwache Anreizstruktur: In allen drei Unternehmen erfolgte die Teilnahme an internem Crowdsourcing auf freiwilliger Basis. Oftmals kann damit eine Teilnah- 
me nicht per Weisung erzwungen werden. Zudem können in der Regel auch keine monetären Anreize gesetzt werden. Da internes Crowdsourcing normalerweise innerhalb der Arbeitszeit stattfindet, würde eine zusätzliche Vergütung der Crowd Worker eine Besserstellung bedeuten, was wahrscheinlich weder von Betriebsräten noch von Führungskräften freigegeben werden würde. Die große Herausforderung liegt darin, dass nur begrenzte Möglichkeiten bestehen, die schwache Anreizstrukturen zu verbessern, da der potenzielle Pool an Teilnehmenden durch die Anzahl der Mitarbeiter eines Unternehmens beschränkt ist.

So stand das Crowdsourcing-Management der „Industrie AG“ beispielsweise vor einer Crowd, die sich darüber beschwerte, die gleichen Aufgaben wiederholt zu erledigen, sich langweilte und darüber nachdachte, die Teilnahme zu beenden. Andere sahen den Nutzen der Initiative auf persönlicher Ebene nicht, priorisierten die tägliche Arbeitsaufgabe höher und beschlossen, sich nicht den internen Crowdsourcing-Initiativen anzuschließen. Da das interne Crowdsourcing-Management keine direkte Autorität hatte, um die Crowd zu motivieren sowie die Abwanderung von Teilnehmern zu verhindern, stand es vor der Herausforderung die Initiativen mit der notwendigen Kapazität erfolgreich durchzuführen.

- 5. Parallelität der Arbeitsabläufe: Die Parallelität der täglichen Arbeit und dem vollständig digital vermittelten Arbeitsumfeld des internen Crowdsourcings kann die Komplexität und den Stress erhöhen. Im Gegensatz zur regulären Arbeit der Crowd Worker, bei der sie direkt mit Kunden oder Kollegen interagieren, ist das interne Crowdsourcing eine herausfordernde Arbeitssituation. Crowd Worker arbeiten allein am Computer, ohne direkte persönliche Interaktion und Austausch. Sie müssen sich in eine dynamische Gemeinschaft von Crowd Workern integrieren, neue digital vermittelte Arbeitsbeziehungen aufbauen und den häufigen impliziten Verhaltenskodex und die Richtlinien in internen Crowdsourcing-Initiativen verinnerlichen. Darüber hinaus können sich die Aufgaben, die bei internem Crowdsourcing durchzuführen sind, im Vergleich zu einem regulären Arbeitsplatz eines Crowd Workers stark unterscheiden und sich ständig ändern.

So hatten die Mitarbeiter der Industrie AG einen ganz anderen Arbeitsalltag als im Crowdsourcing. Dort mussten sie eine mobile App testen, die ihre Kunden auf ihrem Smartphone nutzen. Crowd Worker auf der ganzen Welt testeten diese Anwendung in ihren verschiedenen Sprachversionen auf einer Vielzahl von Smartphones. Für die Ausführung dieser Aufgabe, benötigen die Mitarbeiter ein ruhiges Arbeitsumfeld um sich auf den Testaufwand zu konzentrieren. Die Mitarbeiter benötigten immer eine gewisse Zeit, bis sie in den neuen Arbeitsmodus wechselten. Die Mitarbeiter der Crowd hatten besonders viel Mühe mit dem Umgang, der Bewertung und Dokumentation von Fehlern und Softwareproblemen. Die Suche nach einem geeigneten Standort und die Umstellung auf die neue digitale Arbeitsweise ohne persönlichen Kontakt wurde von jeder zweiten Person als stressig oder eher stressig empfunden. 
Tab. 3 Lösungen zur Bewältigung der Transformationsherausforderungen

\begin{tabular}{|c|c|c|}
\hline Herausforderungen & Lösungen & Beschreibung \\
\hline $\begin{array}{l}\text { 1. Organisatorische } \\
\text { Grenzen }\end{array}$ & $\begin{array}{l}\text { 1. Grünes Licht für } \\
\text { internes Crowdsourcing }\end{array}$ & $\begin{array}{l}\text { Das Crowdsourcing-Management findet Ar- } \\
\text { gumente, um direkte Vorgesetzte davon zu } \\
\text { überzeugen, Mitarbeiter in die Crowd aufzu- } \\
\text { nehmen und während der Arbeitszeit interne } \\
\text { Crowdsourcing-Aufgaben durchzuführen, z. B. } \\
\text { einen angemessenen Zeitraum }\end{array}$ \\
\hline $\begin{array}{l}\text { 2. Gehypte Erwartun- } \\
\text { gen }\end{array}$ & $\begin{array}{l}\text { 2. Klare Erwartungen } \\
\text { im Vorfeld schaffen }\end{array}$ & $\begin{array}{l}\text { Das Management sollte die Erwartungen durch } \\
\text { ein Kommunikationskonzept erfüllen und steu- } \\
\text { ern, dass die Rolle des Crowd Workers und den } \\
\text { Einfluss der Crowds auf die Initiative vor der } \\
\text { ersten internen Crowdsourcing-Beteiligung } \\
\text { deutlich macht }\end{array}$ \\
\hline $\begin{array}{l}\text { 3. Inadäquate IT Fähig- } \\
\text { keiten }\end{array}$ & $\begin{array}{l}\text { 3. Crowd durch Trai- } \\
\text { ning und Unterstützung } \\
\text { befähigen }\end{array}$ & $\begin{array}{l}\text { Das Management muss durch eine angemessene } \\
\text { Schulung und Unterstützung das Mindestmaß } \\
\text { an IT-Fähigkeiten sicherstellen, insbesondere } \\
\text { weil einige Mitarbeiter nicht aus IT-Berufen } \\
\text { stammen }\end{array}$ \\
\hline $\begin{array}{l}\text { 4. Schwache Anreiz- } \\
\text { struktur }\end{array}$ & $\begin{array}{l}\text { 4. Konzept designen, } \\
\text { welches die Crowd } \\
\text { langfristig motiviert }\end{array}$ & $\begin{array}{l}\text { Um der abnehmenden Motivation der Crowd } \\
\text { entgegenzuwirken, sollte das Management ein } \\
\text { Motivationskonzept mit vielen verschiedenen } \\
\text { Anreizmaßnahmen entwickeln }\end{array}$ \\
\hline $\begin{array}{l}\text { 5. Parallelität der Ar- } \\
\text { beitsabläufe }\end{array}$ & $\begin{array}{l}\text { 5. Crowd beim Wechsel } \\
\text { zwischen der Crowd- } \\
\text { sourcing und tradi- } \\
\text { tionellen Arbeitswelt } \\
\text { unterstützen }\end{array}$ & $\begin{array}{l}\text { Einige Mitarbeiter erleben den ständigen Wech- } \\
\text { sel zwischen dem Crowdsourcing und dem tra- } \\
\text { ditionellen Umfeld als stressig und anstrengend. } \\
\text { Als Gegenmaßnahmen bietet dieser Artikel eine } \\
\text { Schaffung ruhiger Arbeitsplätze und Versetzung } \\
\text { von Mitarbeitern }\end{array}$ \\
\hline
\end{tabular}

\section{Lösungsstrategien zur Bewältigung der Herausforderungen}

Auf Basis der Erfahrungen der drei untersuchten Unternehmen werden im Folgenden erfolgreiche Lösungsstrategien skizziert und in Tab. 3 zusammengefasst.

- 1. Grünes Licht für internes Crowdsourcing: Unternehmen müssen für einen organisatorischen Rahmen sorgen, in dem sich die Mitarbeiter bewegen können. Dieser Rahmen muss Konflikten mit Vorgesetzten vorbeugen und Sicherheit für teilnehmende Crowd Worker schaffen. Gleichzeitig muss dieser im Idealfall aber auch deren Vorgesetzte und andere involvierte Stakeholder von den Vorteilen einer Teilnahme ,ihrer“ Mitarbeiter überzeugen. In diesem Kontext hat sich gerade der Kompetenzgewinn der Mitarbeitenden durch die Teilnahme am internen Crowdsourcing als eines der schlagenden Argumente herausgestellt. Zudem ist in dem Rahmen genau abzustecken, wie viel Zeit die Teilnahme am internen Crowdsourcing in Anspruch nehmen darf. Des Weiteren ist vorab genau abzuklären, welche weiteren internen Stakeholder bei einer internen Crowdsourcing-Initiative an Bord geholt werden müssen. Dies kann beispielsweise wie bei der Versicherungen AG den Betriebsrat umfassen. Es ist jedoch anzumerken, dass je nach Unternehmen auch anderen internen Stakeholdern eingebunden werden müssen - so 
waren dies beispielsweise unterschiedliche Regionalgesellschaften, Compliance und die interne Unternehmenskommunikation bei der Bank AG. Auch wenn sich hier keine allgemeingültigen Empfehlungen geben lassen, sollten Unternehmen beachten, dass bei diesen Abklärungen oftmals grundsätzliche Ansichten über die internen Prozesse und Strukturen eines Unternehmens tangiert werden und internes Crowdsourcing nur erfolgreich sein kann, wenn hier ein Konsens zwischen allen Stakeholdern gefunden werden kann.

- 2. Klare Erwartungen schaffen: Eine Lücke zwischen den Erwartungen der Crowd und der Realiät des internen Crowdsourcing kann sowohl die Aktivität als auch die Leistungsfähigkeit einer internen Crowd negativ beeinflussen. Daher sollte das Management von internen Crowdsourcing-Plattformen die Erwartungen durch eine klare Kommunikation mit der Crowd systematisch steuern und dem entstehenden „Hype Cycle“ aus nicht erfüllbaren Anfangserwartungen und Desillusionierung entgegensteuern. Bei der Bank AG hat sich dabei insb. ein zweistufiges Kommunikationskonzept bewährt. In einer ersten Phase sollte die Kommunikation die Rolle des Crowd Workers vor der ersten Teilnahme deutlich machen. Zum Beispiel sollten eine klare Vision und Mission kommuniziert werden, damit Crowd Worker eine Zielvorstellung sowie ein Bild ihrer Rolle als Crowd Worker entwickeln können. In der zweiten Phase sollte sich die Kommunikation auf das Aufrechterhalten der Motivation der Mitarbeiter konzentrieren, z. B., indem sie die Möglichkeit betont, neue Dinge zu lernen, ihnen ein Gefühl der sozialen Integration gibt und proaktiv die Erfolge hervorhebt, die das interne Crowdsourcing bereits erzielen konnte.

- 3. Etablieren von Onboarding-Prozessen: Im Allgemeinen sollten die Crowdsourcing-Plattform und die damit abgebildeten Arbeitsabläufe so einfach und intuitiv wie möglich gestalten werden. Darüber hinaus sollten Crowd Worker vor der Teilnahme an internen Crowdsourcing in Bezug auf ihre Aufgaben, den Umgang mit der Crowdsourcing-Plattform sowie den übergeordneten Arbeitsprozessen geschult werden. Auf Basis der Erfahrungen der untersuchten Unternehmenspartner haben sich dabei differenzierte Schulungsformate als erfolgreich erwiesen. Für viele Crowd Worker eignen sich einfache Schulungsmaterialien, mit denen sich schnell ein Überblick verschafft werden kann. Darüber hinaus sollten aber gewissen Nachschlage-Werke wie Frequently-Asked-Questions (FAQs) angeboten werden, wo die wichtigen und komplizierten Schritte erklärt werden. Ebenso sollte ein gewisser Support für Crowd Worker angeboten werden, der individuelle Fragen bei Bedarf beantwortet. Die Erfahrungen der Bank AG zeigen, dass dies insb. während einzelner Software-Tests essenziell war. So wurde beispielsweise der Crowd vor jedem Test durch einen fachlichen Experten in einem Video Chat erklärt, was genau getestet werden soll, wo dabei die größten Schwierigkeiten zu erwarten sind und wie etwaige Software-Fehler dokumentiert werden sollen. Diese Video Chats dauerten oftmals nur wenige Minuten und bedurften in der Regel keine besondere Vorbereitung. Sie befähigten die Crowd Fragen und Erwartungen vorab zu klären, so dass die Qualität der Ergebnisse deutlich gesteigert und eine Vielzahl, der beim Testen der Software auftretenden Fragen durch andere Crowd Worker beantwortet werden konnte. 
- 4. Unterschiedliche Motive ansteuern: Um die Motivation der Mitarbeiter über die Zeit zu erhalten, sollte eine Vielzahl verschiedener Anreize entwickeln werden. Die Manager von internen Crowdsourcing-Plattformen sollten dabei berücksichtigen, dass ihre Crowd eine mannigfaltige Ansammlung von Individuen ist, die durch verschiedenste Motive angetrieben wird. Grundsätzlich teilen dabei jedoch alle drei untersuchten Unternehmen die Erfahrung, dass das Bilden einer „Community “ den effektivsten Anreizmechanismus darstellt. So versucht die Versicherungen AG beispielsweise, die Motivation zum sozialen Austausch zu fördern, indem sie regelmässig informelle Treffen mit Getränken und Snacks für die Crowd organisiert, um die Kollegen im wirklichen Leben kennenzulernen und Dankbarkeit für die Teilnahme auszudrücken. Diese sozialen Ereignisse sind wichtig, weil sie das Aufbauen persönlicher Beziehung fördern und bei allen beteiligten Stakeholdern ein Gefühl der Verbundenheit schaffen. Durch dieses Gemeinschaftsgefühl konnten die Manager die Bedürfnisse der Crowd verstehen und eine gewisse Loyalität bei den Crowd Workern aufbauen. Darüber hinaus erwiesen sich das Vermitteln des Gefühls neue Kenntnisse erlernt zu haben und die Befriedigung von Neugier als äußerst starke Anreize. Kern ist jedoch in beiden das Stellen interessanter und abwechslungsreicher Aufgaben. Gerade das Streben neue Kenntnisse zu erlernen lässt sich zudem sehr gut mit extrinsischen Anreizen verbinden. Beispielsweise erstellt die Versicherungen AG jährliche Teilnahme-Zertifikate für die Crowd Worker, in dem die Teilnahme an unterschiedlichen Software Testing Aktivitäten dokumentiert wird. Zusätzlich hat sich das interne Crowdsourcing bei der Bank AG als Karrieresprungbrett für besonders geeignete Software Tester etabliert.

- 5. Crowd beim Wechsel zwischen der Crowdsourcing und traditionellen Arbeitswelt unterstützen: Das Management sollte der Crowd Unterstützung bieten. Wenn sich der Crowd Worker darauf konzentrieren kann, die internen CrowdsourcingAufgaben ohne Unterbrechung und mit einem Maß an Stille durchzuführen, nimmt der Stresspegel ab.

Zum Beispiel gehen einige Mitarbeiter von Bank AG in einen ruhigen Raum im Büro, wo niemand den Geräuschpegel erhöht. Andere nutzen Räume, die physisch weit von ihrer täglichen Arbeit entfernt sind, um Unterbrechungen durch ihre Kollegen zu vermeiden oder nutzen Home-Office.

\section{Schlussbemerkungen}

Internes Crowdsourcing ist eine innovative Form der Arbeitsorganisation, die es Unternehmen erlaubt die Agilität und die Effektivität interner Arbeitsprozesse zu erhöhen. Die Einführung von internem Crowdsourcing stellt jedoch für viele Unternehmen einen großen organisatorischen Transformationsprozess dar, den es zu steuern gilt. In diesem Artikel beschreiben wir in drei Fällen, wie Unternehmen dabei mit fünf zentralen Herausforderungen konfrontiert werden und zeigen wie sie erfolgreiche Lösungsansätze einsetzten, um diese zu überwinden. Trotz der herausfordernden Veränderungen in der Arbeitsorganisation integrierten die Unternehmen 
erfolgreich zusammen über 400 Mitarbeiter in einem digitalen Umfeld zwecks Software Testing. Ohne die umfassende Unterstützung der verschiedenen Crowds hätten die Unternehmen die Software-Projekte mangels Ressourcen oder Kompetenzen nicht durchführen können. Lösungen für die spezifischen Probleme zu entwickeln und zu testen brauchte Zeit, Motivation und Geduld aller Beteiligten. Die Leser dieses Artikels können von den Erfahrungen der Fälle profitieren, Herausforderungen voraussehen und bereits frühzeitig Lösungsansätze einsetzen, um Ressourcen zu sparen. Letztendlich haben sich alle drei Unternehmen nach den Erfahrungen aus den Fällen dazu entschlossen auch in Zukunft internes Crowdsourcing zu nutzen oder gar auszubauen, um auch in weiteren Bereichen des Unternehmens die Vorteile dieser neuen Arbeitsorganisation zu nutzen.

\section{Literatur}

Benbya H, Leidner D (2016) Harnessing employee innovation in internal crowdsourcing platforms: lessons from Allianz UK. Vortrag, International Conference on Information Systems (ICIS), Dublin

Creswell JW (1998) Qualitative inquiry and research design: choosing among five traditions, 2. Aufl.

Durward D, Blohm I, Leimeister JM (2016) Crowd work. In: Business \& information systems engineering, S $1-6$

Eisenhardt KM (1989) Building theories from case study research. Acad Manage Rev 14(4):532-550

Kohler T (2015) Crowdsourcing-based business models: how to create and capture value. Calif Manage Rev 57(4):63-84

Leicht N, Blohm I, Leimeister JM (2017) Leveraging the power of the crowd for software testing. IEEE Softw 34(2):62-69

Malhotra A, Majchrzak A (2014) Managing crowds in innovation challenges. Calif Manage Rev 56(4): $103-123$

Rohrbeck R, Thom N, Arnold H (2015) IT tools for foresight: The integrated insight and response system of Deutsche Telekom Innovation Laboratories. Technol Forecast Soc Change 97:115-126

Simula H, Vuori M (2012) Benefits and barriers of crowdsourcing in B2B firms: Generating ideas with internal and external crowds. Int J Innov Manag 16(06):1-19

Steinert M, Leifer L (2010) Scrutinizing Gartner's hype cycle approach. In: Technology Management for Global Economic Growth (PICMET) 2010 Proceedings of PICMET'10. IEEE, Phuket, Thailand, S 1-13

Stieger D, Matzler K, Chatterjee S, Ladstaetter-Fussenegger F (2012) Democratizing strategy: How crowdsourcing can be used for strategy dialogues. Calif Manage Rev 54(4):44-68

Yin R (1994) Case study research: design and methods. SAGE, Beverly Hills

Zetsche D (2017) Daimler 2017: Daimler and the transformation of the automotive industry. Annual Shareholders' Meeting of Daimler AG.

Zuchowski O, Posegga O, Schlagwein D, Fischbach K (2016) Internal crowdsourcing: conceptual framework, structured review, and research agenda. J Inf Technol 31(2):166-184 